\title{
EL CONCEPTO INTERSECCIONALIDAD EN EL FEMINISMO NEGRO DE PATRICIA COLLINS.
}

The Concept of Intersectionality in Patricia Collins's Black Feminism.

\author{
Daniel Santibañez Guerrero ${ }^{1}$ \\ dsantibanezguerrero@gmail.com
}

\begin{abstract}
Resumen
Dentro de las principales corrientes de feminismo político desarrolladas a fines del siglo XX, el feminismo negro constituye con seguridad uno de los intentos filosóficos más exhaustivos en el examen de las bases teóricas del sexismo y la discriminación hacia las mujeres. Dentro de estos planteamientos, el pensamiento de Patricia Collins abordará el nexo histórico y cultural que los conceptos sexo, género y raza presentarían desde la óptica de la "interseccionalidad", entendiendo la discriminación las mujeres negras como una de las formas últimas (sino la mayor) de exclusión y sometimiento del ser humano.
\end{abstract}

Palabras claves: Collins, Feminismo negro, Interseccionalidad, Matriz de dominación.

\begin{abstract}
Within the main currents of political feminism developed in the late twentieth century, black feminism is surely one of the most comprehensive philosophical attempts to examine the theoretical basis of sexism and discrimination against women. Within these approaches, the account of Patricia Collins will address the historical and cultural nexus that the concepts "sex", "gender" and "race" would present from the perspective of "intersectionality", with black women being understood as one of the ultimate forms (if not the greatest) of exclusion and submission of the human being.
\end{abstract}

Keywords: Collins, Black feminism, Intersectionality, Matrix of oppression.

Fecha de Recepción: 13/11/2017 - Fecha de Aceptación: 26/03/2018

${ }^{1}$ Licenciado en Educación/Profesor de Estado en Filosofía y Magíster en Filosofía Política por la Universidad de Santiago de Chile. Doctor (c) en Filosofía -m/ Filosofía Moral y PolíticaUniversidad de Chile, Chile (Becario CONICYT). 


\section{Introducción.}

Patricia Collins (1948), socióloga, pedagoga y académica norteamericana en la Universidad de Maryland, es en la actualidad una de las principales representantes del denominado feminismo negro, corriente de pensamiento que surge en Estados Unidos a fines de la década de los ochenta (extendiéndose al Caribe y América Latina), y que entiende las desigualdades históricas experimentadas por las mujeres de color a partir de un marcado vínculo entre sexismo, discriminación de clases y racismo, el cual formalmente destacará por primera vez Kimberlé Crenshaw (1959) en 1989 a través del concepto "interseccionalidad".

Collins, retomando el término de Crenshaw, examinará en su primer gran trabajo, Black Feminist Thought: Knowledge, Consciousness and the Politics of Empowerment (1990), la constitución de sistemas de poder a partir del nexo entre razaletnia, género, clase social, sexualidad y nación/Estado, categorías mutuamente entrecruzadas que, proyectadas en diferentes capas de la sociedad, ejercen múltiples formas de opresión cuyo blanco central será (particularmente en el contexto social estadounidense) la mujer negra.

En tal sentido, la profunda influencia de los medios de comunicación, aparejado al individualismo que promueve fuertemente la actual sociedad de consumo, de algún modo limitarán la supresión de prácticas discriminatorias hacia la mujer de color, no sólo en los ámbitos de la política, el trabajo y la educación, sino también al interior de los mismos círculos intelectuales. Estos círculos padecerán una suerte de "ceguera" frente a la posición históricamente desfavorable de las mujeres de color, especialmente los movimientos de igualdad racial, quienes concentrarían su discurso en la defensa de la igualdad del "hombre" negro, y los diferentes movimientos feministas cuyas reivindicaciones involucran, en último término, una defensa de la mujer "blanca".

De esta manera, economía, administración política e ideología operan como un sistema de control velado, pero altamente efectivo, diseñado para mantener a las mujeres afroamericanas en el lugar subordinado que les ha sido asignado y donde los medios de comunicación, la academia y el mundo civil aparecen como colaboradores de una discriminación que, al involucrar género, clase y raza, constituye una de las formas más profundas de segregación femenina en el pasado y en la actualidad.

\section{Orígenes y fundamentos del feminismo negro.}

Dentro de las principales corrientes feministas que se desarrollan en la actualidad, el feminismo negro representa con seguridad una de las líneas interpretativas que más acentúan los aspectos sociales y políticos de la postergación de la mujer, 
particularmente en el caso de las mujeres de color que, bajo el punto de vista de las principales representantes de esta corriente, han enfrentado y siguen enfrentando situaciones de discriminación con elementos privativos asociados a su sexo y color de piel.

Los inicios de esta línea feminista, como explica Jabardo (27), se encuentran en la confluencia y tensión de dos movimientos representativos y marcadamente políticos del siglo XIX en Estados Unidos: el abolicionismo y el sufragismo. En el primero de ellos, fundamental en el surgimiento de la posterior Guerra de Secesión (1861-1865), el centro de reclamación será la derogación de la esclavitud de la población negra, institución fuertemente practicada en los estados del sur de Norteamérica como Maryland, Missouri, Delaware y Kentucky (los denominados "estados fronterizos"), en contraste con la prohibición casi mayoritaria de esta institución en los estados del norte. Como sabemos, la derogación oficial de la esclavitud se llevará a efecto a través de la Decimotercera Enmienda a la Constitución de los Estados Unidos en 1865, cuatro años antes de la primera aprobación en el estado de Wyoming del derecho a voto de la mujer, pilar fundamental de la demanda del movimiento sufragista que, impulsado en sus inicios por Susan Anthony (18201906), Lucy Stone (1818-1893) y Elisabeth Cady Stanton (1815-1902), y luego de las sucesivas aprobaciones en Utah (1870), Colorado (1893), Idaho (1896), Washington (1910), California (1911), Oregón, Arizona y Kansas (1912) Nevada y Montana (1914), etc., finalmente logra su objetivo a través de la Decimonovena Enmienda a la Constitución de los Estados Unidos que en 1920 otorgará derecho a sufragio a la mujer.

A pesar de representar sendas defensas de la igualdad racial y de la igualdad de género, como las teóricas del feminismo negro hacen notar, ninguno de estos procesos incorporará ni en la práctica ni en su foco discursivo a las mujeres de color (Jabardo 27). La confluencia de elementos étnicos y sexuales termina por marginarlas y postergarlas del debate, tanto por parte de los movimientos críticos a la segregación racial que surgirán a fines de la década del sesenta (cuyo discurso igualitario se concentraría, en el fondo, en la defensa del "hombre" negro), como por parte de las corrientes feministas que surgen con fuerza a fines de 1970 (que en su defensa de la mujer estarían concentrándose, básicamente, en un modelo de mujer "blanca").

La convergencia entre etnia y sexo que limita las pretensiones de libertad e igualdad de las mujeres negras en el siglo XIX será percibida, no obstante, por parte de algunas emblemáticas mujeres de color, quienes intentarán instalar contra la fuerte represión cultural de la época y el olvido de parte de los movimientos abolicionistas y sufragistas- una defensa de la mujer de color orientada a resaltar, precisamente, su condición de mujer. Así, la conocida abolicionista y activista negra Sojourner Truth (1797-1883) pronuncia su célebre 
discurso Ain't I a Woman (suerte de texto fundacional del feminismo negro) en la Convención de los Derechos de la Mujer en Akron (1852), con un tono y un mensaje muy similar al que posteriormente Ida Wells (1862-1931) manifestara en su autobiográfica Crusade for Justice (1928) y Mary Church Terrell (1863-1954) en A Colored Woman in a White World (1940).

Desde niveles teóricos diferentes (Wells a través de un discurso reflexivo y agudo, Truth desde el testimonio vivaz y potente) las bases del feminismo negro que estas autoras establecen lo perfilarán como una corriente de pensamiento en la que el examen teórico se concibe aparejado a la movilización, y donde la forma de generación de pensamiento se entiende de manera colectiva y contra hegemónica. Y es que a diferencia del "feminismo blanco" que se adscribe a un ejercicio de racionalidad propio de la Ilustración, a través de figuras como Olympe de Gouges (1748-1793) y especialmente Mary Wollstoncraft (1759-1797), el feminismo negro al emerger en el contexto del esclavismo - adopta una posición de reflexión mucho más inclusiva, intentado la incorporación de diversos saberes, pensamientos, lógicas y actores sociales, y no sólo la asimilación y reproducción de un modelo de racionalidad uniforme como el presente en la Ilustración (Jabardo 28).

Desde sus inicios, el feminismo negro tomará distancia, pues, del modelo de razón instrumental que la modernidad funda, sustentándose en un ejercicio de oralidad que intenta instalar un relato más que un discurso y emprendiendo de manera precursora una labor de deconstrucción, de re-significación del concepto hegemónico "mujer" (Jabardo 29).

\section{El concepto "interseccionalidad".}

El nexo entre raza y género, abordado por las iniciadoras del feminismo negro a fines del siglo XIX, aparece como uno de los conceptos centrales del pensamiento de Patricia Hill Collins, una de las principales referentes del feminismo negro (en su vertiente estadounidense) en la actualidad.

Collins, junto con el trabajo de Audre Lorde (1934-1992), Angela Davis (1944), Alice Walker (1944), Paula Giddings (1947), Patricia Bell-Scott (1950), Bell Hooks (1952) y Kimberlé Williams Crenshaw (1959), entre otras, viene a representar el resurgimiento del enfoque feminista negro que emerge en la segunda mitad del siglo XIX, tanto en lo referido a sus elementos metodológicos (relato, oralidad, etc.), como a su trasfondo conceptual (Viveros 3). En el caso de Collins, este resurgimiento tendrá su eje central - como las mismas estudiosas negras tienden a coincidir- en la noción de "interseccionalidad", concepto que originalmente establece Kimberlé Crenshaw a fines de la década de los ochenta, pero que Collins adopta y reinterpreta en la línea de una lectura de mayor trasfondo filosófico. 
Los antecedentes del concepto interseccionalidad, como bien explica Viveros (3), tienen sus orígenes precisamente en la primera tradición de feministas ilustradas ("feminismo blanco") de mediados del siglo XVIII, y en las ya destacadas feministas negras afroamericanas de mediados del siglo XIX. En este punto, por ejemplo, intentado abordar la interconexión de los elementos que componen las diferentes modalidades de dominación, Olympe de Gouges en Francia planteará la conexión género-esclavitud, mientras (como vimos) Sojourner Truth lo hará con el nexo sexo-raza (Ibid.). En ambos casos, no obstante, el ejercicio teórico se realizará sin una definición conceptual exacta del procedimiento metodológico realizado.

Ya adentrados en el último tercio del siglo XX, en específico 1989, Kimberlé Crenshaw (de formación en Derecho) acuñará formalmente el término interseccionalidad en el marco de su desempeño como abogada defensora de un grupo de trabajadoras negras de la compañía General Motors, con el objetivo de resaltar la invisibilidad jurídica de las múltiples dimensiones de opresión que estas mujeres recibían por parte de la gerencia de la compañía (Crenshaw 141). Se tratará, como la misma Crenshaw ha resaltado, de un concepto cuyo uso se ha empleado de manera contextual y práctica, enfocado principalmente para el análisis de omisiones jurídicas y desigualdades específicas, y no con la pretensión directa de erigir una teoría de la dominación femenino-racial (concepción teórica frente a la cual Crenshaw, sin embargo, no manifiesta ni un rechazo ni una oposición).

La recepción del concepto por parte de Collins en Black Feminist Thought: Knowledge, Consciousness and the Politics of Empowerment (1990), no obstante, sí se enmarcará dentro del proyecto de elaboración de una reflexión en torno a la articulación de mecanismos de dominación de las mujeres de color, siendo la primera autora en referirse a la interseccionalidad ya como un paradigma (Viveros 4). Este paradigma, como sostiene Barriteau (7), iría en función directa de una de las denuncias centrales de las teóricas negras: la ineficacia de los marcos explicativos vigentes (tanto en el feminismo tradicional como en los movimientos de igualdad racial) para comprender y explicar la realidad y los fundamentos teóricos de la exclusión y discriminación de las mujeres negras.

\section{La interpretación de Collins del concepto "interseccionalidad".}

El concepto interseccionalidad, como varios estudiosos han resaltado, presenta algunos importantes e interesantes puntos de discrepancia entre las teóricas. Algunos de estos focos de debate se centrarán en aspectos "estructurales", donde Kathy Davis considerará que el intento de sistematización del enfoque por parte de, por ejemplo, Ange Marie Hancock en Intersectionality as a normative and 
empirical paradigm (2007)2, no necesariamente representa un avance para la epistemología del feminismo negro, tomando en cuenta el origen histórico y acento que este ejercicio reflexivo tiene en el testimonio y el relato (Viveros 6). Por otra parte, en lo referido a los niveles en los cuales el análisis interseccional debe ser desarrollado, Dorthe Staunæs sostendrá que éste debe desarrollarse en las dimensiones subjetivas de las relaciones de poder (muy en sintonía con los enfoques estructuralistas y postmodernos), mientras que para Patricia Collins el análisis deberá circunscribirse a las estructuras sociales, distanciándose un poco del excesivo recurso del examen de narración de identidades (Ibid.). Esta diferencia en las opiniones da cuenta, en último término, de lo que Viveros denomina la "doble afiliación teórica y genealógica" que la interseccionalidad presenta: el black feminist y las corrientes postmodernas/posestructuralistas (Ibid.).

A pesar de estas diferencias, Collins destaca cómo en torno a los márgenes generales del concepto sí existe un consenso general y claro, lo que permitiría hacer referencia a éste, básicamente, de la siguiente manera:

The term intersectionality references the critical insight that race, class, gender, sexuality, ethnicity, nation, ability, and age operate not as unitary, mutually exclusive entities, but as reciprocally constructing phenomena that in turn shape complex social inequalities (2015 2).

De ser así, como la misma autora explica, la interseccionalidad podría conceptualizarse como un proyecto de conocimiento global cuyos contornos cambiantes se desarrollan en línea con las formaciones sociales de desigualdades complejas. Su riqueza conceptual (y en gran medida filosófica, bajo nuestro punto de vista) se encuentra entonces precisamente en estos márgenes generales amplios desde los cuales encara el examen de la articulación social de las desigualdades, y no en el uso de categorías rígidas expresadas en definiciones específicas y propias de un ejercicio reflexivo instrumental (Collins 2015 5).

En este sentido, el concepto de interseccionalidad que Collins presenta le permite desde el punto de vista metodológico abordar la relación entre género y raza como elementos de un sistema social de opresión donde, intersectados entre sí en la figura de la mujer de color, la opresión ejercida adquiere una estructura y una fuerza

\footnotetext{
${ }^{2}$ Hancock entiende la interseccionalidad como un paradigma que conforma una teoría normativa y una investigación empírica. En tal sentido, la autora identifica seis supuestos básicos del concepto que, grosso modo, apuntan a considerar que: 1) en todo problema y proceso político complejo se involucran más de una categoría de diferencia; 2) se debe prestar atención a todas las categorías pertinentes y, especialmente, a las relaciones entre categorías (éstas son variables); 3) cada categoría es diversa internamente; 4) las categorías de diferencia se conceptualizan y abordan como producciones dinámicas de factores individuales e institucionales; 5) la investigación interseccional examina las categorías a varios niveles y en las interacciones entre estos; 6) la interseccionalidad como paradigma requiere de un estudio tanto teórico como empírico (Hancock 248-254).
} 
mayor a la presente en la opresión del hombre negro y la mujer blanca. Este carácter interconectado, no obstante, será entendido como propio y característico de los sistemas de opresión, los cuales en la práctica no operan a través de focos aislados de dominación (esto es, sólo desde la clase, sólo desde la raza o sólo desde el género), sino más bien desde la conformación de categorías híbridas como clase-etnia, clase-raza, clase-género, etc. (Collins 2000 18). En tal sentido, y como la misma autora señala, al exponer en un lugar protagónico específicamente las experiencias de opresión de las mujeres estadounidenses de color, su propuesta explicativa de la articulación y ejecución de las estructuras sociales de opresión no se aplicarán únicamente al caso de las mujeres negras: sitúa la historia y los relatos de postergación de mujeres negras en el centro de su análisis sin pretender con ello, en definitiva, privilegiar esos relatos de postergación (Id. 228).

Complementando los elementos que posibilitarían una aproximación más profunda a los fundamentos de la exclusión de la mujer de color, se vuelve indispensable para Collins un examen tanto microsociológico como macrosociológico. Esta diferencia, de acuerdo a la socióloga norteamericana, sustenta y se expresa en una distinción léxica que se plasma en dos términos primordiales de su pensamiento:

a) La noción propiamente de "intersectionality", referida a formas particulares de opresión, formas específicas ejercidas desde la conexión de categorías.

b) La noción de "matrix of oppression", la cual se vincula con la organización estructural de las categorías que permiten el ejercicio práctico de la opresión (Collins 2000 18).

Así, en la lectura de Collins, la dominación de la mujer de color no da cuenta de la existencia de una visión cultural (ideología, paradigma, tradición, etc.) desde la cual emergen las formas específicas de dominación. Antes bien, estas formas específicas de dominación surgirían desde una organización social e institucional ("matriz de dominación") donde, en el caso de la historia de la discriminación de la mujer negra en Estados Unidos, escuelas, universidades, vivienda, empleo, salud, gobierno y otras instituciones sociales operan opresivamente conformando una superestrctura rígida, pero de formas y modelos cambiantes (Collins 2000 227, 228).

Por este motivo, al citar el ejemplo de Senegal, Estados Unidos e Inglaterra, donde las respectivas feministas negras de cada país coinciden en denunciar un sistema opresivo que mezcla raza, género, clase y sexualidad, Collins resalta la forma distinta en que en cada una de estas naciones dicha opresión toma su forma concreta. Así:

(...) regardless of how any given matrix is actually organized either across time or from society to society, the concept of a matrix of domination encapsulates the universality of intersecting oppressions as organized through diverse local realities (Collins 2000 228). 
De este modo, cuatro serían los ámbitos de dominios de poder que, desde su intersección, ejecutan la opresión hacía la mujer de color: estructural, disciplinario, hegemónico e interpersonal (Collins 2000 299). Con ello, los procesos sociales de opresión (que por las características bosquejadas hasta este punto adquieren claramente dimensiones políticas, económicas e ideológicas) operarán desde ámbitos mucho mayores al del sólo sometimiento físico de la persona: afectarán también directamente -desde la generación de un conocimiento que se erige a través de su organización institucional- la autodefinición y autopercepción de aquellos sujetos oprimidos a fin de mantenerlos -como en el caso de la mujer estadounidense de color- en un lugar subordinado de explotación, donde medios de comunicación, academia y mundo civil han colaboran con su sujeción tanto de forma activa como pasiva.

\section{Conclusiones.}

Desde el punto de vista filosófico, es importante resaltar que la defensa de la mujer negra por parte de Collins, junto con involucrar un claro componente ético y político, también comprende una epistemología en tanto involucra una deconstrucción del concepto de "mujer negra" $\mathrm{y}$, especialmente, de los mecanismos de sometimientos de la misma.

Dentro de esta óptica, si bien la presencia del concepto "interseccionalidad" le entrega a este ejercicio reflexivo un marco conceptual importante, la lectura de Collins no desarticula la riqueza del elemento testimonial que, como hemos presenciado, conforma el eje del análisis que el feminismo negro realiza de la opresión de la mujer de color. Una epistemología "alternativa", en palabras de Jabardo (35), se constituiría entonces a través del examen de una historia de opresión y de la conservación de la misma a través del relato, contrastándose así con el empleo de un lenguaje técnico, objetivo y distante de su objeto de reflexión, propio de una concepción de la filosofía, la racionalidad y la misma condición humana que vinculará su origen con los inicios de la modernidad europea.

En este sentido, la producción del conocimiento que el feminismo negro desarrolla (particularmente en la visión de Collins) entrega a dicho conocimiento una carga valórica sustancial al encontrarse vinculado -en el relato de esta historia que se piensa- con una emoción y vitalidad muy en sintonía con varias líneas de pensamiento contemporáneo, complementando así la fuerza de su reclamo con el anhelo reivindicatorio que se erige. Con ello, en un nexo filosófico que no sólo se encontrará en parte de lo metodológico, sino también en la declaración de una intención transformadora de la realidad, el feminismo negro adopta un compromiso ético fuerte y afín con la tradición marxista, al proponerse precisamente la 
modificación de las estructuras opresivas que (en este caso) afectan a la mujer de color.

El relato desde el cual el feminismo negro manifiesta su examen y reclamo frente a la opresión, involucra de este modo un diálogo con las diferentes corrientes filosóficas de su época, comunicación que desde su lectura crítica se aproxima tanto a la ilustración, al vitalismo, al marxismo y al existencialismo, como a la hermenéutica y al posestructuralismo, constituyéndose de tal modo en una reflexión que mira a su interior desde la proyección de su lectura al mundo.

Por este motivo, la interpretación feminista negra que Collins representa tanto desde su concepción de interseccionalidad que permite interpretar el nexo entre raza y género, como desde su visión de la articulación y constitución de superestructuras de opresión que, por sus características, operan desde aristas interconectadas- no sólo involucra una lectura de la opresión, de la mujer o de la lógica y la mecánica de dominación, sino también finalmente una mirada sobre la condición humana misma que, también moldeada desde estas superestructuras, en algunos casos omite el actuar de estos mecanismos de opresión sobre ciertos grupos humanos, mientras que en otros los avala e incluso participa de forma activa y directa.

\section{Bibliografía.}

Barriteau, Violet Eudine. “Aportaciones del feminismo negro al pensamiento feminista: una perspectiva caribeña”, Boletín ECOS 14 (2011): 1-17.

Collins, Patricia. Black Feminist Thought. New York: Routledge, 2000.

Collins, Patricia. "Intersectionality's Definitional Dilemmas", Annual Review of Sociology 41 (2015): 1-20.

Collins, Patricia. “La política del pensamiento feminista negro". Qué son los estudios de mujeres. Marysa Navarro y Catharine Stimpson, editores. Buenos Aires: FCE, 1998: 253-312.

Crenshaw, Kimberlé. "Demarginalizing the Intersection of Race and Sex: A Black Feminist Critique of Antidiscrimination Doctrine, Feminist Theory and Antiracist Politics", University of Chicago Legal Forum 1 (1989): 139-167.

Hancock, Ange-Marie. "Intersectionality as a normative and empirical paradigm", Politics and Gender 3/2 (2007): 248-254.

Jabardo, Mercedes. Feminismos negros. Una antología. Madrid: Traficante de Sueños, 2012. 
Viveros, María. "La interseccionalidad: una aproximación situada a la dominación", Debates feministas 52 (2016): 1-17. 